\title{
Monocyte-to-Lymphocyte Ratio is Associated with Depression 3 Months After Stroke
}

This article was published in the following Dove Press journal:

Neuropsychiatric Disease and Treatment

\section{Kai-qi Ding* \\ Ze-hua Lai* \\ Yu Zhang \\ Guo-yuan Yang \\ Ji-rong $\mathrm{He}$ \\ Li-li Zeng}

Department of Neurology and Institute of Neurology, Ruijin Hospital/Luwan Branch, Shanghai Jiao Tong University School of Medicine, Shanghai, People's Republic of China

*These authors contributed equally to this work
Correspondence: Li-li Zeng

No. 197 Ruijin Second Road, Huangpu

District, Shanghai, 200025, People's

Republic of China

Tel +86-13816290607

Email Ilzeng@126.com

ji-rong $\mathrm{He}$

No. 149 Chongqing South Road, Huangpu

District, Shanghai, People's Republic of

China

Tel +86-13162762045

Email kyo3shao@aliyun.com
Purpose: To explore the relationship between the monocyte-to-lymphocyte ratio (MLR) and depression three months after acute ischemic stroke.

Patients and Methods: From May 2013 to September 2014, 203 patients with acute ischemic stroke were recruited within 7 days post-stroke from Shanghai Ruijin Hospital and blood samples were collected after admission. The Hamilton Depression Scale and Clinical Review were evaluated at 3 months after stroke. Based on the Diagnostic and Statistical Manual of Mental Disorders-IV diagnostic criteria, we divided patients into post-stroke depression (PSD) and nonPSD groups. We analyzed the intergroup difference in MLR and the contributing factors. Moreover, dynamic changes in monocytes, lymphocytes and MLR at four different time intervals for all the stroke patients and their relationship with PSD patients were also studied.

Results: The NIHSS scores and MLR in the PSD group were significantly higher than in the non-PSD group $(\mathrm{p}<0.05)$. Logistic regression analysis revealed MLR was an independent risk factor for PSD (odds ratio: 18.020, 95\% confidence interval: 1.127-288.195, $\mathrm{p}=0.041$ ). MLR correlated negatively with cholesterol and low-density lipoprotein $(\mathrm{r}=-0.160$ and -0.165 , respectively, $\mathrm{p}<0.05$ ). Within 7 days post-acute ischemic stroke, monocytes gradually increased while lymphocytes remained unchanged for all the stroke patients. The MLR value was significantly higher in the PSD group than in the non-PSD group within $24 \mathrm{~h}$ post-stroke $(\mathrm{p}<0.05)$, but there was no difference in the other three time-intervals between the two groups. Conclusion: The admission MLR, particularly within $24 \mathrm{~h}$ post-stroke, was associated with PSD at 3 months, implying that the MLR might be involved in the PSD inflammatory mechanism.

Keywords: MLR, depression, inflammation, acute ischemic stroke

\section{Introduction}

Stroke is commonly encountered in the neurology department. Depression is a common complication after stroke. ${ }^{1}$ The stroke recurrence rate and disability rate of patients with post-stroke depression (PSD) are significantly higher than in those without depression, which greatly affects rehabilitation and quality of daily life. ${ }^{2,3}$ At present, the pathogenesis of PSD is multifactorial and unclear but appears to involve neurobiological dysfunction caused by cerebral ischemia, influenced by psychosocial distress and neurobiological dysfunctions. ${ }^{4,5}$

After the occurrence of ischemic stroke, blood perfusion is insufficient, and thus the oxygen and glucose needed for brain tissue metabolism are inadequate. ${ }^{6}$ Extensive nerve necrosis and release of a large number of cytokines and chemokines trigger an inflammatory cascade and cause destruction of the blood-brain barrier (BBB). ${ }^{7}$ In addition, upregulation of neutrophils and monocytes and the reduction of lymphocytes 
were immediately induced in the peripheral blood after stroke. ${ }^{8}$ They were attracted by the inflammatory mediators and recruited and infiltrated to the brain through the damaged $\mathrm{BBB}$, further aggravating the inflammatory damage to ischemic brain. $^{4}$

Some studies have shown that the occurrence of depression is related to inflammation in the brain. ${ }^{9,10}$ Proinflammatory factors affect neurotransmitters or neural circuits and change the level of serotonin (5-hydroxytryptamine, 5-HT) and norepinephrine. ${ }^{11,12}$ Additionally, inflammatory factors can also activate the stress response system. ${ }^{13}$ For example, hyperactivity of the hypothalamus-pituitary-adrenal axis (HPA) leads to disorder of the neuroendocrine and sympathetic nervous system, which is related to the genesis and development of depression. ${ }^{9,11}$, The stress response of patients with depression is higher and in an over-activation state than in individuals without depression. ${ }^{12,14}$

The damaged BBB after stroke promotes the entry of peripheral immune cells into the brain, which greatly contributes to the occurrence and development of PSD. For example, it has been reported that the neutrophil-tolymphocyte ratio (NLR) and peripheral inflammatory markers, such as C-reactive protein, platelet-to-lymphocyte ratio (PLR) and interleukin-6, are increased in PSD patients. ${ }^{15,16}$ Other studies have found that the MLR is also an independent risk factor for cardiovascular disease, and can reflect the severity of the disease. ${ }^{17,18}$ For example, the higher MLR value of ischemic stroke patients is related to the degree of carotid artery stenosis and it is an independent risk factor for carotid artery stenosis. ${ }^{19}$ It is also associated with stroke-related pneumonia (SAP), which is helpful for clinicians to establish an early diagnosis for patients with high-risk SAP. ${ }^{20}$ Moreover, studies have found MLR values to be elevated in patients with affective disorders or schizophrenia. ${ }^{21,22}$

Other studies have shown that the lymphocyte-tomonocyte ratio (LMR) is related to the prognosis of cardiovascular disease and tumors. ${ }^{23}$ For instance, LMR is associated with poor prognosis 3 months after stroke, ${ }^{24}$ which may be due to the LMR value that not only indicates inflammation response but also represents the degree of immunosuppression. ${ }^{25,26}$ Nevertheless, there has been no research about the correlation between the monocyte-to -lymphocyte ratio (MLR) and PSD, and their relationship is still unclear.

Monocytes increased gradually within 7 days after stroke, and lymphocytes are mainly inactivated within 12 hours after stroke and reach the lowest point after
$48 \mathrm{~h} .{ }^{27}$ Monocytes can differentiate into macrophages and dendritic cells, which are the main cells involved in innate immunity, ${ }^{28}$ while lymphocytes mainly play an important role in specific immunity. Therefore, these cells mediate two different immune or inflammatory pathways and provide better information than single leukocyte parameters. ${ }^{29}$

We hypothesized that investigation of the relationship between MLR and PSD may facilitate an understanding of the inflammatory mechanism in the pathogenesis of PSD. In this study, we investigated the MLR in patients with acute ischemic stroke within 7 days after the attack and analyzed its association with depression at 3 months post-stroke as well as its dynamic changes after stroke.

\section{Patients and Methods Study Subjects}

From May 2013 to September 2014, we recruited 203 consecutive patients with acute ischemic stroke within 7 days of stroke onset, from the Department of Neurology, Shanghai Jiaotong University School of Medicine affiliated Ruijin Hospital (Shanghai, China). This study was approved by the ethics committee of Shanghai Jiao Tong University School of Medicine affiliated Ruijin Hospital. All the patients provided informed consent, and this study was conducted in accordance with the Declaration of Helsinki.

The inclusion criteria were as follows: (1) meeting the diagnostic criteria of acute ischemic stroke based on clinical symptoms and imaging examinations, (2) willingness to complete a depression scale evaluation 3 months after stroke, and (3) less than 1 week since stroke onset.

The following patients were excluded: (1) patients with infection and inflammatory diseases; (2) patients with aphasia and cognitive impairment; (3) patients with failure of important organs, such as heart, liver, and kidney; (4) patients with a history of mental illnesses, such as depression, anxiety, and schizophrenia; and (5) patients with tumors or autoimmune diseases.

\section{Clinical Evaluation and Data Collection}

Patients were recorded the time of stroke onset, and they underwent routine blood tests, computed tomography, or magnetic resonance imaging prior to medication and completed the National Institute of Health Stroke Scale assessment at admission to the Emergency Department. We also 
recorded the past medical history and medications of the stroke patients. After entering the Neurology Department, the routine blood tests were performed again, and we kept a record of the specific time of detecting blood routine. There were other related laboratory and imaging examinations were used to confirm the etiology of stroke, including blood glucose and blood lipid levels, carotid Doppler ultrasonography, computed tomography angiography (CTA) or magnetic resonance angiography (MRA), electrocardiography (ECG), and echocardiography. The Trial of Organization in Acute Stroke Treatment (TOAST) and Oxfordshire Community Stroke Project (OCSP) classifications were determined by two neurologists based on the patient's medical history and auxiliary tests. All patients were treated according to the Chinese Guidelines for the Prevention of Ischemic Stroke and Transient Ischemic Attack. ${ }^{30}$ After 3 months, we performed the Hamilton Depression Scale during a clinical interview to evaluate their depression symptoms.

We also recorded the following information: gender, age, education level (high school and above), blood pressure (systolic pressure/diastolic pressure), blood glucose, smoking, drinking, atrial fibrillation, medications (including anti-platelet, anti-coagulant, non-steroid antiinflammatory, anti-lipemic and disease-modifying antirheumatic drugs), hypertension, diabetes, hyperlipidemia, heart disease, previous strokes, NIHSS score, TOAST classification, OCSP classification, carotid artery stenosis, and intracranial artery stenosis. In addition, triglyceride, total cholesterol, high-density lipoprotein (HDL), lowdensity lipoprotein (LDL), apolipoprotein A (APOA) and APOB levels were determined. The diagnostic criteria of hypertension, diabetes and hyperlipidemia were described in our previous study. ${ }^{31}$

\section{Blood Sample Collection}

We recruited patients with acute ischemic stroke within 7 days of stroke onset. Prior to medication administration, $4 \mathrm{~mL}$ of cubital venous blood was drawn for blood routine tests in the emergency department. Some patients agreed to blood sampling again after entering the neurology ward and we recorded every time patients did blood routine. In total, 203 patients with acute ischemic stroke were enrolled in this study, and their times of stroke onset were recorded. We performed the first blood routine test before medication in the Emergency Department, and most patients agreed to do the second test on the next day. Therefore, there are 314 observations for 203 patients. According to times when peripheral blood samples were collected after poststroke, these 203 acute ischemic stroke patients were divided into four groups: <24 h, 24-48 h, 48-72 h, and $>72 \mathrm{~h}$. The monocytes, lymphocytes, and MLR values were recorded and analyzed.

\section{Diagnosis of Depression}

According to the Diagnostic and Statistical Manual for Mental Disorders-IV criteria, the diagnosis of PSD was based on a clinical interview and the Hamilton Depression Scale-17 evaluation. The questionnaire contained 17 items, each of which is scored on a 5-point scale ( 0 is none, 1 is suspicious or mild, 2 is mild, 3 is moderate, and 4 is severe). Two raters evaluated patients independently. If the total score was $\leq 7$, patients were considered nondepressed, and when the score was $>7$ points and the depressive symptoms had lasted more than 2 weeks, patients were considered to have PSD.

\section{Statistical Analysis}

We used SPSS 22.0 statistical software (IBM Inc., Armonk, NY) to process the data. The KolmogorovSmirnov test was used to assess the normality of data distribution. The two independent samples $t$-test was applied to analyze measurement data with a normal distribution, which were expressed as mean \pm standard deviation $(\overline{\mathrm{x}} \pm \mathrm{s})$. Enumeration data were analyzed by means of the $\chi 2$ test and were shown as percentage or rate. We used the Mann-Whitney $U$-test and the Kruskal-Wallis test for data with a non-normal distribution, with results presented as median and interquartile range [M ( $\mathrm{P}$ 25, $\mathrm{P}$ 75)]. Logistic regression analysis was used to evaluate the factors influencing PSD, and receiver operating characteristic (ROC) curve analysis was performed to evaluate the MLR in PSD patients. According to interquartile range of MLR for all patients, we divide them into 5 groups, Q1, Q2, Q3, Q4 and all. The correlation between MLR and other factors was analyzed using Spearman correlation analysis and we draw the scatter diagram of related variables and fit the line in the diagram. To assess dynamic changes in monocytes, lymphocytes, and the MLR in patients, we used the Kruskal-Wallis test, and the Mann-Whitney $U$-test was used to analyze differences between the two groups. Statistical significance was defined as $\mathrm{p}<0.05$. 


\section{Results}

\section{General Characteristics of Post-Stroke Depression (PSD) and Non-PSD Groups}

We screened 320 consecutive patients with acute ischemic stroke. Among them, 69 patients did not meet the inclusion criteria, and 29 patients had no blood routine data. In the subsequent 3-month follow-up period, one patient died, two patients had recurrent stroke, 15 cases were lost to follow-up, and one patient had serious systemic disease. Therefore, only 203 patients were included in this study, 44 of whom were in the PSD group and 159 were in the non-PSD group (Figure 1).

In both groups, there was no significant difference in sex or age between the two groups. All patients enrolled were of Han nationality from mainland China. However, in the PSD patient group $(\mathrm{n}=44)$, the MLR, NIHSS score and blood glucose were significantly higher than those in the non-PSD patient group $(n=159, p<0.05)$. There was no obvious difference between the two groups in terms of education years, blood pressure (systolic pressure/diastolic pressure), smoking, drinking, atrial fibrillation, medications (anti-platelet, anti-coagulant, non-steroid anti-inflammatory, anti-lipemic and disease-modifying anti-rheumatic drugs), hypertension, hyperlipidemia, cardiac disease, previous strokes, TOAST classification, OPCA classification, carotid artery stenosis, intracranial artery stenosis, triglyceride, total cholesterol, high-density lipoprotein (HDL), low-density lipoprotein (LDL), apolipoprotein A (APO-A), apolipoprotein B (APO-B), lymphocyte, or monocyte values (Table 1).

\section{MLR at Admission is Associated with PSD at 3 Months}

We incorporated factors with $\mathrm{p}<0.1$ into multivariate analysis in logistic regression analysis, and the result showed that MLR was an independent risk factor for PSD (odds ratio, OR: 18.020, 95\% confidence interval, CI: 1.127-288.195, $\mathrm{p}=0.041$ ) (Table 2). The area under the ROC curve was $0.615(\mathrm{p}=0.020,95 \%$ CI: 0.524 0.705). The cut-off of MLR value for predicting PSD was 0.2298 , yielding a sensitivity and specificity of $77.3 \%$ and $54.1 \%$, respectively (Figure 2 ).

\section{Cholesterol and Low-Density Lipoprotein are Negatively Correlated with MLR}

According to the interquartile range of the MLR, we divided the patients into five groups, separately Q1

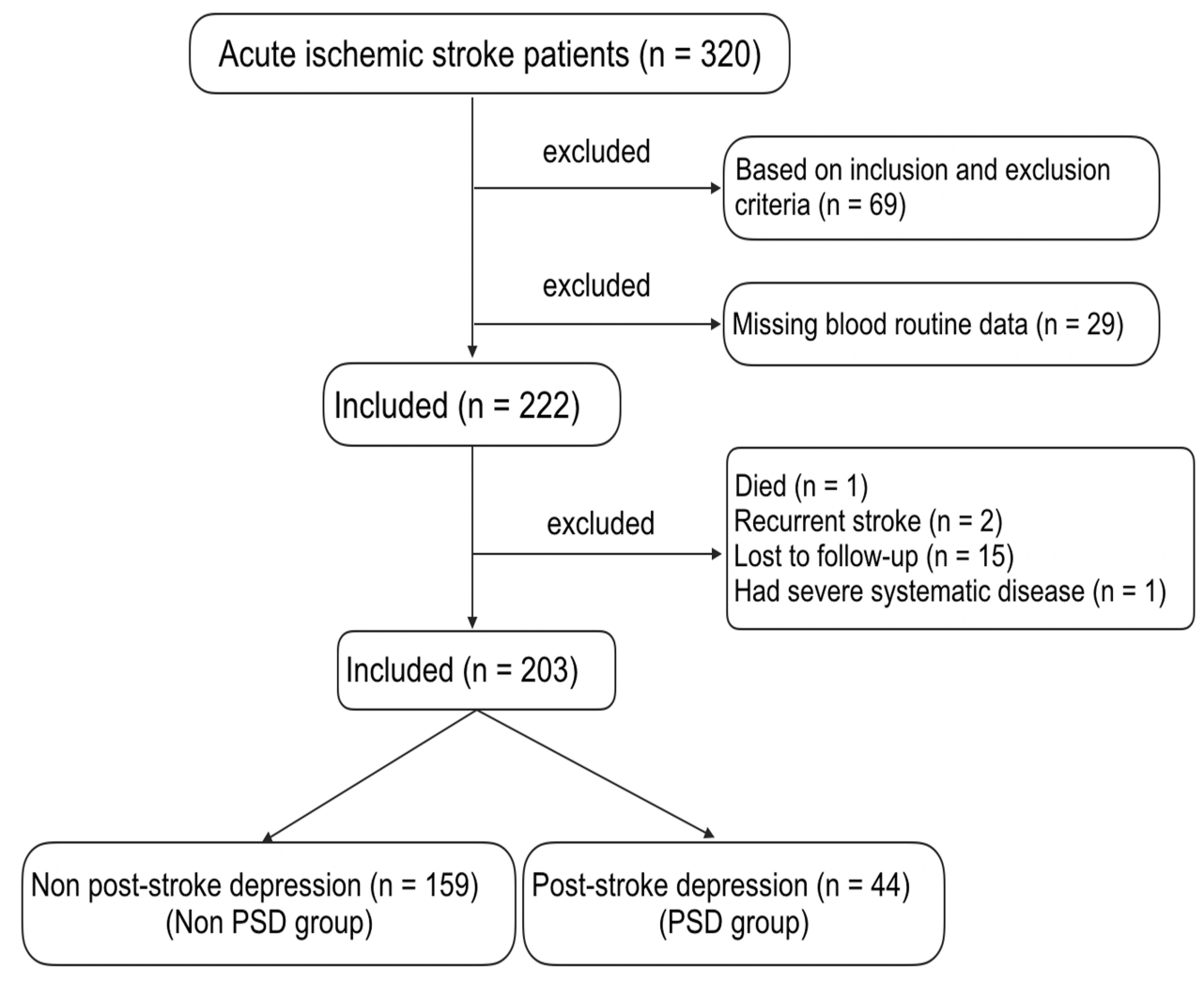

Figure I The recruitment of patients with acute ischemic stroke in this study. 
Table I General Characteristic Analysis of Patients in the PSD Group and Non-PSD Group

\begin{tabular}{|c|c|c|c|}
\hline Variables & PSD Group $(n=44)$ & Non-PSD Group $(n=159)$ & $P$ value \\
\hline Sex (male) & $30(68.2 \%)$ & $108(67.9 \%)$ & 0.564 \\
\hline Age (year) & $68.11 \pm 8.73$ & $66.16 \pm 11.15$ & 0.273 \\
\hline Education (> 12 years) & $22(50.0 \%)$ & 85 (53.5\%) & 0.685 \\
\hline Hypertension & $35(79.5 \%)$ & $120(75.5 \%)$ & 0.574 \\
\hline Systolic pressure $(\mathrm{mmHg})$ & $148.28 \pm 24.17$ & $148.89 \pm 23.52$ & 0.87 \\
\hline Diastolic pressure $(\mathrm{mmHg})$ & $84 \pm 11.79$ & $87.63 \pm 46.78$ & 0.89 \\
\hline Blood glucose & $6.97 \pm 2.74$ & $5.99 \pm 2.13$ & $0.032 *$ \\
\hline Diabetes & $20(45.5 \%)$ & $48(30.2 \%)$ & 0.058 \\
\hline Hyperlipidemia & $20(45.5 \%)$ & $74(46.5 \%)$ & 0.518 \\
\hline Cardiac disease & $6(13.6 \%)$ & 30 (18.9\%) & 0.423 \\
\hline Atrial fibrillation & I (2.3\%) & $9(5.7 \%)$ & 0.345 \\
\hline Previous stroke times & $0(0,0.75)$ & $0(0,0)$ & 0.227 \\
\hline Smoking & $17(38.6 \%)$ & $50(31.4 \%)$ & 0.43 \\
\hline Drinking & $10(22.7 \%)$ & 31 (I9.5\%) & 0.69 \\
\hline \multicolumn{4}{|l|}{ Medications } \\
\hline Anti-platelet & $26(59.1 \%)$ & $98(61.6 \%)$ & 0.618 \\
\hline Anti-coagulant drug & $0(0 \%)$ & $0(0 \%)$ & 1.00 \\
\hline Anti-lipemic drug & $0(0 \%)$ & $3(1.9 \%)$ & 0.35 \\
\hline NIHSS score & $4(2-6.75)$ & $2(1-4)$ & $0.001 *$ \\
\hline TOAST classification & & & 0.484 \\
\hline LAA & $21(47.7 \%)$ & $83(52.2 \%)$ & \\
\hline CE & I (2.3\%) & $6(3.8 \%)$ & \\
\hline SAO & $13(29.5 \%)$ & $44(27.7 \%)$ & \\
\hline SOD & $0(0.0 \%)$ & I $(0.6 \%)$ & \\
\hline SUD & $9(20.5 \%)$ & $25(I 5.7 \%)$ & \\
\hline OPCA classification & & & 0.702 \\
\hline $\mathrm{PACl}$ & $21(47.7 \%)$ & $80(50.3 \%)$ & \\
\hline $\mathrm{POCl}$ & $17(38.6 \%)$ & $40(25.2 \%)$ & \\
\hline LACI & $6(13.6 \%)$ & $39(24.5 \%)$ & \\
\hline Carotid artery stenosis & $26(59.1 \%)$ & $83(52.2 \%)$ & 0.333 \\
\hline Intracranial artery stenosis & $22(50.0 \%)$ & $80(50.3 \%)$ & 0.735 \\
\hline Triglyceride(mmol/L) & $1.76 \pm 0.80$ & $1.90 \pm 1.03$ & 0.585 \\
\hline Total cholesterol $(\mathrm{mmol} / \mathrm{L})$ & $4.62 \pm 1.25$ & $4.79 \pm 1.08$ & 0.267 \\
\hline High-density lipoprotein(mmol/L) & $1.10 \pm 0.29$ & $1.08 \pm 0.28$ & 0.630 \\
\hline Low-density lipoprotein(mmol/L) & $2.87 \pm 1.08$ & $3.00 \pm 0.91$ & 0.246 \\
\hline Apolipoprotein A (APO-A) (g/L) & $1.17 \pm 0.23$ & $1.19 \pm 0.20$ & 0.610 \\
\hline Apolipoprotein B (APO-B) (g/L) & $0.99 \pm 0.30$ & $1.02 \pm 0.25$ & 0.290 \\
\hline Lymphocyte(109/L) & $1.70 \pm 0.58$ & $|.88 \pm 0.7|$ & 0.147 \\
\hline
\end{tabular}

(Continued) 
Table I (Continued).

\begin{tabular}{|c|c|c|c|}
\hline Variables & PSD Group $(n=44)$ & Non-PSD Group $(n=159)$ & $P$ value \\
\hline Monocyte( $\left(10^{9} / \mathrm{L}\right)$ & $0.48 \pm 0.19$ & $0.45 \pm 0.17$ & 0.178 \\
\hline MLR (monocyte/lymphocyte) & $0.27(0.23-0.33)$ & $0.24(0.18-0.30)$ & $0.020^{*}$ \\
\hline $\mathrm{QI}(\mathrm{Q} I \leq 0.1934)$ & $6(13.6 \%)$ & $48(30.2 \%)$ & $0.028^{*}$ \\
\hline $\mathrm{Q} 2(0.1934<\mathrm{Q} 2 \leq 0.2485)$ & $8(18.2 \%)$ & $33(20.8 \%)$ & 0.707 \\
\hline $\mathrm{Q} 3(0.2485<\mathrm{Q} 3 \leq 0.3077)$ & $16(36.4 \%)$ & $39(24.5 \%)$ & 0.119 \\
\hline $\mathrm{Q} 4(\mathrm{Q} 4>0.3077)$ & $14(31.8 \%)$ & $39(24.5 \%)$ & 0.331 \\
\hline The time between stroke attack and blood routine detecting (hours) & PSD group & Non-PSD group & \\
\hline$<24 \mathrm{~h}$ post-stroke (hours) & $12.47 \pm 7.12$ & $12.49 \pm 6.89$ & 0.99 \\
\hline 24-48 h post-stroke (hours) & $37.97 \pm 7.85$ & $37.22 \pm 6.70$ & 0.67 \\
\hline $48-72$ h post-stroke (hours) & $57.99 \pm 7.15$ & $59.18 \pm 7.53$ & 0.66 \\
\hline$>72 \mathrm{~h}$ post-stroke (hours) & $214.81 \pm 226.27$ & $170.69 \pm 168.07$ & 0.20 \\
\hline
\end{tabular}

Notes: the patients were divided into two groups: PSD and non-PSD groups; QI, Q2, Q3 and Q4 of MLR were the interquartile range of MLR: separately, *p<0.05. Values are expressed as frequency(percentage), mean \pm standard deviation or median \pm interquartile range.

Abbreviations: PSD, post-stroke depression; NIHSS, National Institute of Health Stroke Scale; TOAST, Trial of Organization in Acute Stroke Treatment; LAA, large artery atherosclerosis; CE, cardiac embolism; SAO, small artery occlusion; SOD, stroke of other determined etiology; SUD, stroke of undetermined etiology; OPCA, Oxfordshire Community Stroke Project; PACI, partial anterior circulation cerebral infarction; POCl, posterior circulation cerebral infarction; LACI, lacunar cerebral infarction; MLR, monocyte-to-lymphocyte ratio.

Table 2 Logistic Regression Analysis of Clinical Predictors of PSD

\begin{tabular}{|l|c|c|c|c|c|c|}
\hline Variable & B Value & S.E. Value & Wald Value & P value & OR & $\mathbf{9 5 \%}$ Cl \\
\hline Diabetes & 0.450 & 0.370 & 1.474 & 0.225 & 1.568 & $0.759-3.240$ \\
NIHSS & 0.133 & 0.056 & 5.728 & 0.017 & 1.142 & $1.024-1.274$ \\
MLR & 2.892 & 1.414 & 4.179 & $0.04 I^{*}$ & 18.020 & $1.127-288.195$ \\
Constant & -3.603 & 1.305 & 7.628 & 0.006 & 0.027 & \\
\hline
\end{tabular}

Note: ${ }^{*} p<0.05$

Abbreviations: PSD, post-stroke depression; NIHSS, National Institute of Health Stroke Scale; MLR, monocyte-to-lymphocyte ratio; OR, odds ratio; CI, confidence interval.

$\leq 0.1934,0.1934<\mathrm{Q} 2 \leq 0.2485,0.2485<\mathrm{Q} 3 \leq$ $0.3077, \mathrm{Q} 4>0.3077$ and All the 203 patients. We analyzed the correlation between MLR values and other risk factors, such as related diseases and blood lipid levels. We found that the MLR value of all the 203 patients correlated negatively with total cholesterol and LDL values, with correlation coefficients $(\mathrm{R})$ of -0.160 and -0.165 , respectively $\quad(\mathrm{p}<0.05)$ (Supplemental Table 1). Patients with higher MLR values had relatively lower cholesterol and LDL levels; Two scatter diagrams between MLR and cholesterol, MLR and LDL according to the Spearman correlation analysis are shown in Figure 3.

However, MLR did not correlate with hypertension, diabetes, hyperlipidemia, heart disease, NIHSS score, carotid artery stenosis, intracranial artery stenosis, HDL, APOA, or APOB.

\section{Dynamic Changes in MLR at Different Times After Acute Ischemic Stroke}

We obtained 314 blood routine test results from the 203 stroke patients within 7 days after stroke and divided them into four groups on the basis of when the blood routines were performed after stroke, respectively $<24$ hours, 24-48 hours, 48-72 hours and $>72$ hours. After analyzing the monocytes, lymphocytes, and MLRs at these four different time intervals, compared with $<24 \mathrm{~h}$ after stroke, monocytes showed a slow upward trend than the other time groups.

Monocyte levels in the $24-48 \mathrm{~h}(0.50 \pm 0.20), 48-$ $72 \mathrm{~h}(0.50 \pm 0.17)$, and $>72 \mathrm{~h}(0.48 \pm 0.16)$ were significantly higher than those in the $<24 \mathrm{~h}$ group $(0.43 \pm 0.67) \quad(\mathrm{p}<0.05) \quad($ Figure $4 \mathrm{~A})$. There was no significant difference in lymphocytes within 7 days after stroke $(1.80 \pm 0.67,1.83 \pm 0.69,1.75 \pm 0.74$, 


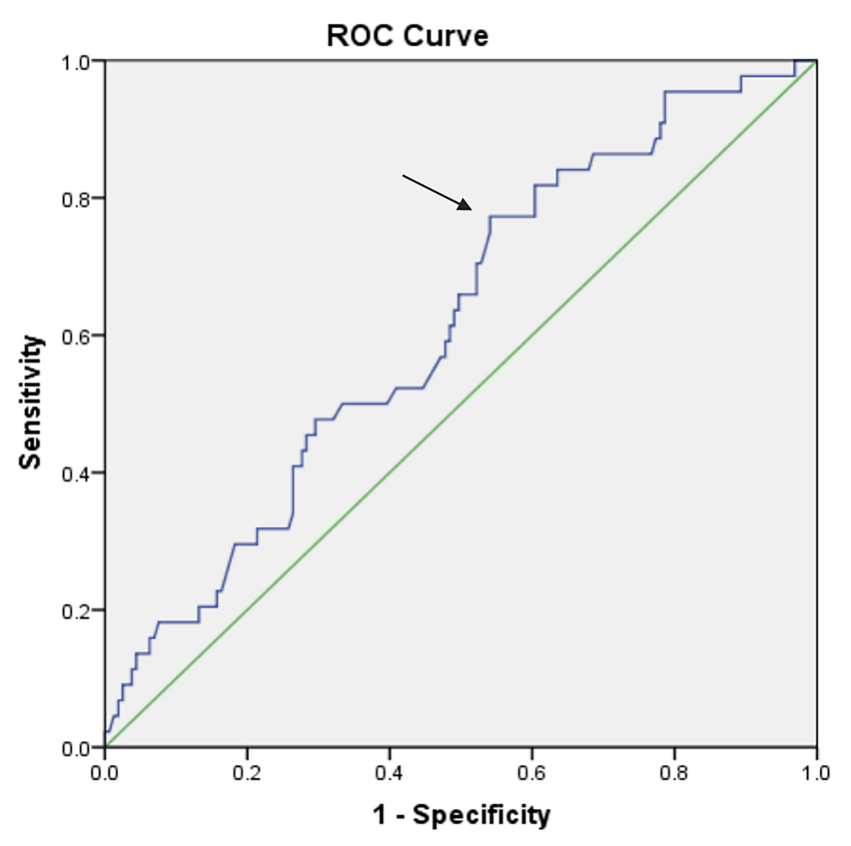

Figure 2 The receiver operating characteristic (ROC) curve of the monocyte-tolymphocyte ratio (MLR) in 203 patients with acute ischemic stroke. The arrow represents the cut-off value.

$1.74 \pm 0.60$, respectively) (Figure 4B). In the $<24$ $\mathrm{h}$ group, the MLR values were the lowest, the MLR values of the 24-48 $\mathrm{h}$ group were higher than those of the $<24 \mathrm{~h}$ group, but there was no statistical difference in the three groups $24-48 \mathrm{~h}, 48-72 \mathrm{~h}$, and $>72 \mathrm{~h}(0.24$ [0.19-0.30], 0.28 [0.19-0.37], $0.25[0.22-0.34]$, and 0.28 [0.21-0.34], respectively; Figure 4C).

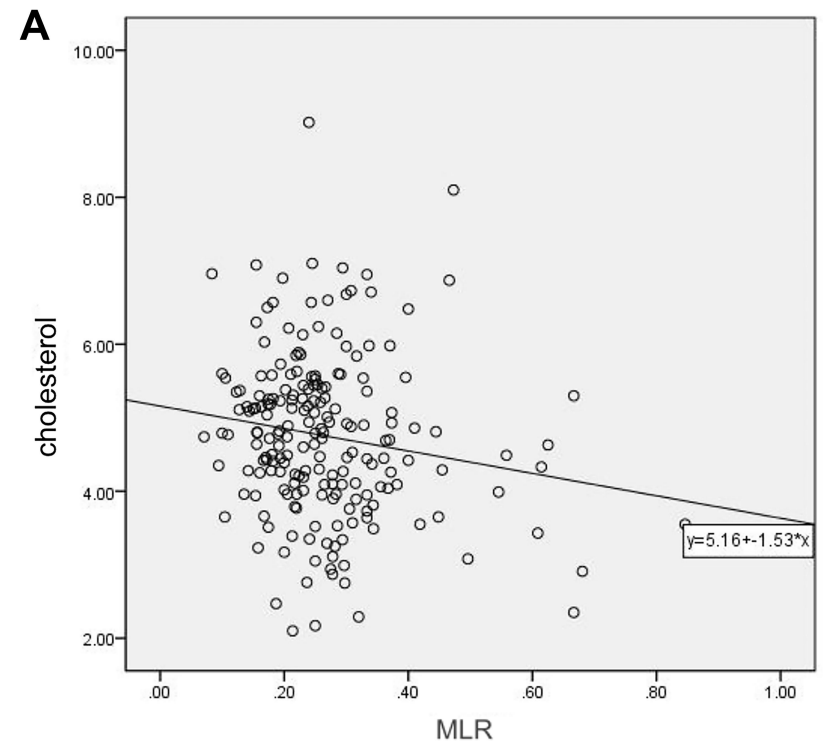

\section{MLR Within $24 \mathrm{~h}$ is Related to PSD}

According to depression symptoms and the Hamilton Depression Scale assessment, we divided all the 203 patients into the PSD group and the non-PSD group and based on the different time-points of collecting blood samples after stroke, we divided the patients into four groups, separately $<24$ hours, 24-48 hours, 48-72 hours and $>72$ hours. In the PSD group, the MLR values in the PSD group were significantly higher than in the non-PSD group within $24 \mathrm{~h}$ after stroke $(\mathrm{p}<0.05)$. There was no significant difference between the PSD and nonPSD groups at other time intervals (Table 3).

\section{Discussion}

The relationship between the MLR and PSD has not been reported to date. In this study, we found that the MLR at admission, particularly the MLR within $24 \mathrm{~h}$ (in the acute phase) is an independent risk factor for depression by 3 months after stroke, which suggests that the MLR may be involved in the pathogenesis of depression after stroke.

In addition to social and psychological factors, biochemical factors also play an important role in the pathogenesis of PSD. At present, studies are increasingly focusing on the relationship between inflammation and mood disorders. For example, cytokines, chemokines, and C-reactive protein levels are higher in patients with depression. ${ }^{32,33}$ These studies suggested that inflammatory markers may be involved in the pathogenesis of depression.

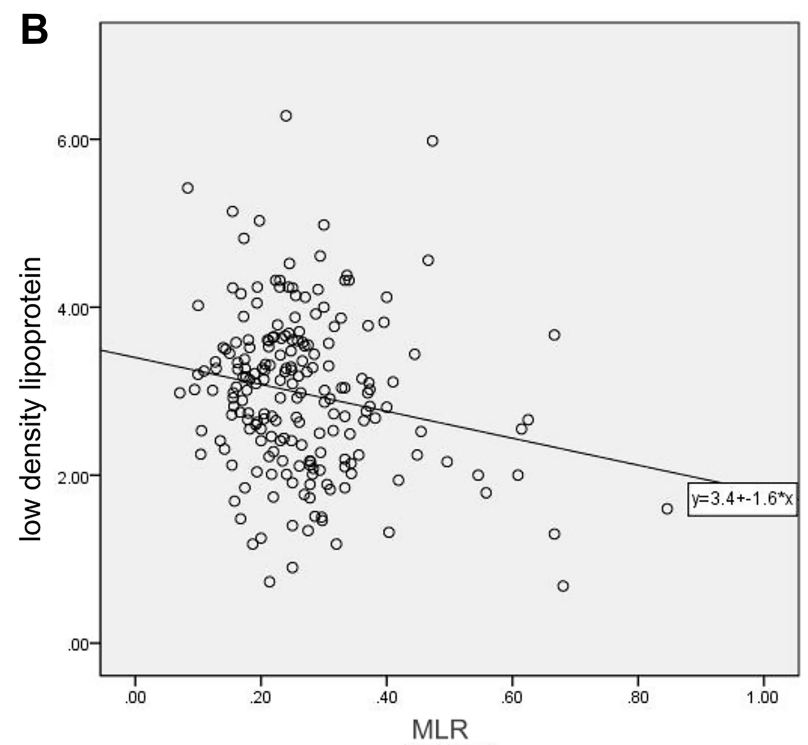

Figure 3 The Spearman correlation analysis of the monocyte-to-lymphocyte ratio (MLR) with cholesterol and low-density lipoprotein (LDL) in all the 203 patients. (A) The relationship between MLR and total cholesterol $(p=0.024, r=-0.160)$. (B) The relationship between $M L R$ and $L D L(p=0.019, r=-0.165)$. 

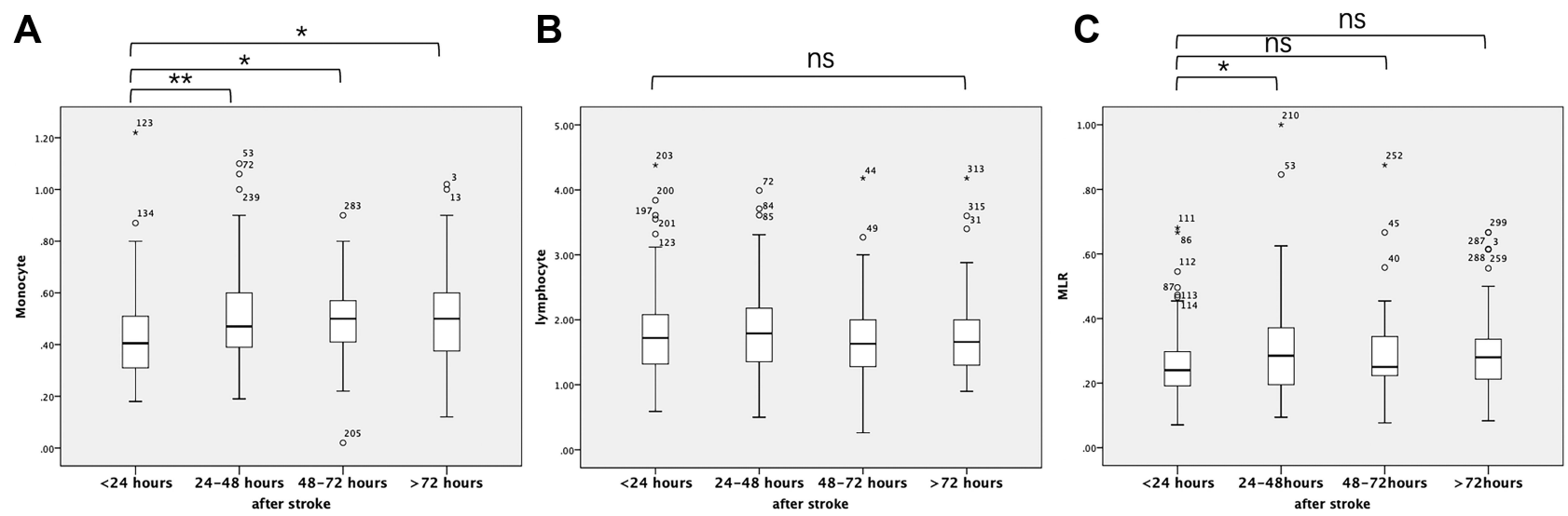

Figure 4 The changes in monocytes, lymphocytes and monocyte-to-lymphocyte ratio (MLR) values in all the 203 patients with acute ischemic stroke at different times after stroke: $<24 \mathrm{~h}, 24-48 \mathrm{~h}, 48-72 \mathrm{~h}$, and $>72 \mathrm{~h}$, respectively. ${ }^{*}<<0.05$, ${ }^{*} \mathrm{p}<<0.0 \mathrm{l}$, ns means no statistical difference. (A) The change of monocyte levels in patients at different times after stroke. (B) The change of lymphocyte levels in patients at different times after stroke. (C) The change of MLR values in patients at different times after stroke.

Stroke, including acute ischemic stroke and intracerebral hemorrhage, can cause a large number of nerve cell deaths and release damage-related molecular patterns (DAMPs). ${ }^{34}$ Local inflammation in damaged brain regions causes secondary brain damage by aggravating bloodbrain barrier destruction, microvascular failure, cerebral edema, oxidative stress and neuronal death. ${ }^{35}$ An increasing amount of evidence has shown that, except for local inflammation, the inflammatory response also occurs in the whole brain, which may continue to affect the patients' long-term neurological function. ${ }^{13}$ The primary injury of stroke triggers a series of events, including the release of excitotoxic substances, oxidative stress and mitochondrial disorders that then causes secondary brain damage, including compression of brain tissue, abnormal blood coagulation, and intracellular biochemical cascade reaction and inflammation. ${ }^{36,37}$ Stroke triggers a series of inflammatory response and activates the immune response. ${ }^{38}$

Microglia in the central nervous system are one of the first cell groups to respond to these dangerous signals. Within a few minutes after injury, microglia are activated, undergo morphological changes, and secrete cytokines. ${ }^{39}$ Some studies have shown that the recruitment and activation of monocytes can enhance the inflammatory reaction and play a role similar to that of the microglia, promoting pro-inflammatory factor and chemokine expression. ${ }^{40,41}$

Table 3 The Comparison of Monocytes, Lymphocytes, and the MLR Between the PSD Group and Non-PSD Group at Different Time Points After Stroke

\begin{tabular}{|c|c|c|c|c|}
\hline \multicolumn{2}{|c|}{ Variables of Different Time } & \multirow{4}{*}{$\begin{array}{c}\text { PSD Group } \\
0.44 \pm 0.16(n=24) \\
1.62 \pm 0.60(n=24) \\
0.25(0.23-0.33)(n=24)\end{array}$} & \multirow{4}{*}{$\begin{array}{c}\text { Non-PSD Group } \\
0.42 \pm 0.16(n=93) \\
1.85 \pm 0.68(n=93) \\
0.23(0.18-0.28)(n=93)\end{array}$} & \multirow{4}{*}{\begin{tabular}{|c|} 
P value \\
0.587 \\
0.096 \\
$0.048^{*}$
\end{tabular}} \\
\hline$<24$ h post-stroke* & Monocyte & & & \\
\hline & Lymphocyte & & & \\
\hline & MLR & & & \\
\hline \multirow[t]{3}{*}{ 24-28 h post-stroke } & Monocyte & $0.53 \pm 0.23(n=15)$ & $0.49 \pm 0.19(n=61)$ & 0.492 \\
\hline & Lymphocyte & $1.59 \pm 0.55(n=15)$ & $1.88 \pm 0.71(n=61)$ & 0.113 \\
\hline & MLR & $0.32(0.20-0.44)(n=15)$ & $0.27(0.19-0.37)(n=61)$ & 0.217 \\
\hline \multirow[t]{3}{*}{ 48-72 h post-stroke } & Monocyte & $0.46 \pm 0.06(n=7)$ & $0.53 \pm 0.16(n=26)$ & 0.304 \\
\hline & Lymphocyte & $1.66 \pm 0.44(n=7)$ & $1.83 \pm 0.76(n=26)$ & 0.947 \\
\hline & MLR & $0.25(0.23-0.33)(n=7)$ & $0.37(0.22-0.37)(n=26)$ & 0.965 \\
\hline \multirow[t]{3}{*}{$>72 \mathrm{~h}$ post-stroke } & Monocyte & $0.49 \pm 0.16(n=21)$ & $0.48 \pm 0.17(n=67)$ & 0.768 \\
\hline & Lymphocyte & $1.71 \pm 0.50(n=21)$ & $1.75 \pm 0.63(n=67)$ & 0.977 \\
\hline & MLR & $0.30(0.24-0.34)(n=21)$ & $0.26(0.20-0.33)(n=67)$ & 0.323 \\
\hline
\end{tabular}

Notes: Values are expressed as mean \pm standard deviation, median and interquartile range. ${ }^{*} \mathrm{p}<0.05$.

Abbreviations: PSD, post-stroke depression; MLR, monocyte-to-lymphocyte ratio. 
We found that the MLR negatively correlated with blood lipids. Patients with higher MLR values have relatively low cholesterol and LDL levels. Many studies have found that depression is related to low blood lipid levels, and that a decrease in total serum cholesterol is related to depression and higher suicide rates. ${ }^{42,43}$ This may be associated with the cholesterol content of the cell membrane, which affects the serotonin transporter, and a decrease in the levels of 5-HT, which is a neurotransmitter that plays an important role in the development of depression. However, hyperlipidemia is considered to be a recognized risk factor for cardiovascular and cerebrovascular diseases. Patients with hyperlipidemia are at high risk for stroke. Other studies have found that hypercholesterolemia is associated with depression. ${ }^{44,45}$ These somewhat contradictory studies reflect the complex relationship among lipids, depression and stroke, especially post-stroke depression. ${ }^{46,47}$

Previous studies comparing a healthy control group and stroke patients found that the number of monocytes in stroke patients increased within 7 days, reached a peak at about 7 days after stroke, and then decreased gradually. While the lymphocytes were largely inactivated and decreased within $24 \mathrm{~h}$ after stroke, they reached the lowest level at about 48 $\mathrm{h}$ after stroke. ${ }^{27,48}$ Therefore, the increase in monocytes and the inactivation of lymphocytes within $48 \mathrm{~h}$ after stroke leads to an increase in the MLR ratio. In our study, compared with the $<24 \mathrm{~h}$ group, monocytes also showed a gradual upward trend at later time points, and the MLR value was the highest at 24-48 h. This was consistent with previous studies. However, when we analyzed the MLR between the PSD and non-PSD groups, the MLR values differed significantly only within $24 \mathrm{~h}$ after stroke, which may be due to the small number of sample cases. Lymphocytes showed no obvious differences across the four different time intervals, which may be due to the earlier inactivation of lymphocytes.

This study had a few limitations. The number of cases in our study was relatively small. We studied the dynamic changes in monocytes, lymphocytes, and the MLR at four different time intervals, but lacked individual comparisons at different times before stroke and after stroke. It is a pity that we did not record body mass index (BMI), individual diet, post-discharge care, or family income of PSD patients, which were both the confounding factors for depression and blood lipids. In addition, this is a 3-month follow-up study, if it had been extended to 6-9 months, the research would have been more accurate and credible. Moreover, monocytes can be divided into three subtypes: classical, intermediate, and non-classical, ${ }^{28}$ while lymphocytes can also be divided into $\mathrm{T}$ and $\mathrm{B}$ types. Such subdivision into specific subtypes may better reveal the role of monocytes in the pathological process of depression.

In conclusion, we show that the admission MLR, particularly within $24 \mathrm{~h}$ post-stroke, was associated with PSD at 3 months, implying that the MLR might be involved in the PSD inflammatory mechanism. The value of the MLR can be obtained from the patient's blood routine tests, quickly and easily, and at low cost, making it of great significance in guiding diagnosis and treatment.

\section{Conclusion}

In the PSD group, the MLR and NIHSS scores were significantly higher than those in the non-PSD group. After some confounding factors were excluded, the result of logistic regression analysis indicated that MLR was an independent risk factor for PSD. Further, patients with higher MLR values had relatively lower cholesterol and LDL levels. Compared with $<24 \mathrm{~h}$ after stroke, monocytes showed a slow upward trend in the other time groups. The admission MLR, particularly within $24 \mathrm{~h}$ post-stroke, was associated with PSD at 3 months, implying that the MLR might be involved in the PSD inflammatory mechanism.

\section{Ethics Approval and Informed Consent}

This study was approved by the ethics committee of Shanghai Ruijin Hospital. All the patients provided informed consent, and this study was conducted in accordance with the Declaration of Helsinki.

\section{Acknowledgments}

We would like to thank the National Natural Science Foundation of China (NSFC) providing funding to L-L Z (project 81471246) and the Hospital specialist development plan of health system in Huangpu District of Shanghai [grant number 2019pyzk01]. We would like to thank Editage (www.editage.cn) for English language editing.

\section{Author Contributions}

All authors made substantial contributions to conception and design, acquisition of data, or analysis and interpretation of data; took part in drafting the article or revising it critically for important intellectual content; agreed to submit to the current journal; gave final approval of the version to be published; and agree to be accountable for all aspects of the work. 


\section{Funding}

This work was supported by the National Natural Science Foundation of China (NSFC) project 81471246 (Li-Li Zeng) and the Hospital specialist development plan of health system in Huangpu District of Shanghai [grant number 2019pyzk01].

\section{Disclosure}

The authors report no conflicts of interest in this work.

\section{References}

1. Altieri M, Maestrini I, Mercurio A, et al. Depression after minor stroke: prevalence and predictors: depression, dysthymia, and minor stroke. Eur J Neurol. 2012;19(3):517-521. doi:10.1111/j.14681331.2011.03583.x

2. Aben I, Verhey F, Lousberg R, Lodder J, Honig A. Validity of the beck depression inventory, hospital anxiety and depression scale, SCL-90, and Hamilton depression rating scale as screening instruments for depression in stroke patients. Psychosomatics. 2002;43 (5):386-393. doi:10.1176/appi.psy.43.5.386

3. Limampai P, Wongsrithep W, Kuptniratsaikul V. Depression after stroke at 12-month follow-up: a multicenter study. Int J Neurosci. 2017;127(10):887-892. doi:10.1080/00207454.2016.1277344

4. Fann DY-W, Lee S-Y, Manzanero S, Chunduri P, Sobey CG, Arumugam TV. Pathogenesis of acute stroke and the role of inflammasomes. Ageing Res Rev. 2013;12(4):941-966. doi:10.1016/ j.arr.2013.09.004

5. Villa RF, Ferrari F, Moretti A. Post-stroke depression: mechanisms and pharmacological treatment. Pharmacol Ther. 2018;184:131-144. doi:10.1016/j.pharmthera.2017.11.005

6. Rodrigo R, Fernández-Gajardo R, Gutiérrez R, et al. Oxidative stress and pathophysiology of ischemic stroke: novel therapeutic opportunities. CNS Neurol Disord Drug Targets. 2013;12 (5):698-714. doi:10.2174/1871527311312050015

7. Dragu R, Huri S, Zukermann R, et al. Predictive value of white blood cell subtypes for long-term outcome following myocardial infarction. Atherosclerosis. 2008;196(1):405-412. doi:10.1016/j.atheroscle rosis.2006.11.022

8. Kim E, Cho S. Microglia and monocyte-derived macrophages in stroke. Neurotherapeutics. 2016;13(4):702-718. doi:10.1007/ s13311-016-0463-1

9. Tiller JWG. Depression and anxiety. Med J Aust. 2013;199(S6):S28S31. doi:10.5694/mja12.10628

10. Ayerbe L, Ayis S, Crichton S, Wolfe CDA, Rudd AG. The natural history of depression up to 15 years after stroke: the South London stroke register. Stroke. 2013;44(4):1105-1110. doi:10.1161/ STROKEAHA.111.679340

11. Ménard C, Hodes GE, Russo SJ. Pathogenesis of depression: insights from human and rodent studies. Neuroscience. 2016;321:138-162. doi:10.1016/j.neuroscience.2015.05.053

12. Long J, Ouyang Y, Duan H, et al. Multiple factor analysis of depression and/or anxiety in patients with acute exacerbation chronic obstructive pulmonary disease. COPD. 2020;15:1449-1464. doi:10.2147/COPD.S245842

13. Shi K, Tian D-C, Li Z-G, Ducruet AF, Lawton MT, Shi F-D. Global brain inflammation in stroke. Lancet Neurol. 2019;18 (11):1058-1066. doi:10.1016/S1474-4422(19)30078-X

14. Tang $\mathrm{Y}, \mathrm{Xu} \mathrm{H}, \mathrm{Du} \mathrm{X}$, et al. Gene expression in blood changes rapidly in neutrophils and monocytes after ischemic stroke in humans: a microarray study. J Cereb Blood Flow Metab. 2006;26 (8):1089-1102. doi:10.1038/sj.jcbfm.9600264
15. Hu J, Zhou W, Zhou Z, Han J, Dong W. Elevated neutrophil-tolymphocyte and platelet-to-lymphocyte ratios predict post-stroke depression with acute ischemic stroke. Exp Ther Med. 2020;19 (4):2497-2504. doi:10.3892/etm.2020.8514

16. Leonard BE. Inflammation and depression: a causal or coincidental link to the pathophysiology? Acta Neuropsychiatr. 2018;30(1):1-16. doi:10.1017/neu.2016.69

17. Ji H, Li Y, Fan Z, et al. Monocyte/lymphocyte ratio predicts the severity of coronary artery disease: a syntax score assessment. BMC Cardiovasc Disord. 2017;17(1):90. doi:10.1186/s12872-017-0507-4

18. Xiang F, Chen R, Cao X, et al. Monocyte/lymphocyte ratio as a better predictor of cardiovascular and all-cause mortality in hemodialysis patients: a prospective cohort study. Hemodial Int. 2018;22(1):82-92. doi:10.1111/hdi.12549

19. Zuo B, Zhu S, Meng X, Zhao D, Zhang J. Monocyte/lymphocyte ratio is associated with carotid stenosis in ischemic stroke: a retrospective analysis. Brain Behav. 2019;9(10):e01429. doi:10.1002/brb3.1429

20. Cheng H-R, Song J-Y, Zhang Y-N, et al. High monocyte-tolymphocyte ratio is associated with stroke-associated pneumonia. Front Neurol. 2020;11:575809. doi:10.3389/fneur.2020.575809

21. Aktaş Karabay E, Demir D, Aksu Çerman A. Evaluation of monocyte to high-density lipoprotein ratio, lymphocytes, monocytes, and platelets in psoriasis. An Bras Dermatol. 2020;95(1):40-45. doi:10.1016/j. abd.2019.05.002

22. Mazza MG, Lucchi S, Rossetti A, Clerici M. Neutrophil-lymphocyte ratio, monocyte-lymphocyte ratio and platelet-lymphocyte ratio in non-affective psychosis: a meta-analysis and systematic review. World J Biol Psychiatry. 2020;21(5):326-338. doi:10.1080/ 15622975.2019.1583371

23. Wu Q, Hu T, Zheng E, Deng X, Wang Z. Prognostic role of the lymphocyte-to-monocyte ratio in colorectal cancer: an up-to-date meta-analysis. Medicine. 2017;96(22):e7051. doi:10.1097/ MD.0000000000007051

24. Ren H, Liu X, Wang L, Gao Y. Lymphocyte-to-monocyte ratio: a novel predictor of the prognosis of acute ischemic stroke. J Stroke Cerebrovasc Dis. 2017;26(11):2595-2602. doi:10.1016/j. jstrokecerebrovasdis.2017.06.019

25. Park M-G, Kim M-K, Chae S-H, Kim H-K, Han J, Park K-P. Lymphocyte-to-monocyte ratio on day 7 is associated with outcomes in acute ischemic stroke. Neurol Sci. 2018;39(2):243-249. doi:10.1007/s10072-017-3163-7

26. Tham T, Olson C, Khaymovich J, Herman SW, Costantino PD. The lymphocyte-to-monocyte ratio as a prognostic indicator in head and neck cancer: a systematic review and meta-analysis. Eur Arch Otorhinolaryngol. 2018;275(7):1663-1670. doi:10.1007/s00405-018-4972-x

27. Vogelgesang A, Grunwald U, Langner S, et al. Analysis of lymphocyte subsets in patients with stroke and their influence on infection after stroke. Stroke. 2008;39(1):237-241. doi:10.1161/ STROKEAHA.107.493635

28. Wang G, Xia Z. Monocyte subsets and their differentiation tendency after burn injury. Front Med. 2013;7(4):397-400. doi:10.1007/ s11684-013-0298-7

29. Matveeva VG, Grigoriev EV. Problems and prospects of investigating monocyte subsets during the development of inflammation-associated diseases. Patol Fiziol Eksp Ter. 2016;60(4):154-159.

30. Wang Y, Liu M, Pu C. 2014 Chinese guidelines for secondary prevention of ischemic stroke and transient ischemic attack. Int J Stroke. 2017;12(3):302-320. doi:10.1177/1747493017694391

31. Zhang Y, He J, Liang H, et al. Diabetes mellitus is associated with late-onset post-stroke depression. J Affect Disord. 2017;221:222-226. doi:10.1016/j.jad.2017.06.045

32. Horsdal HT, Köhler-Forsberg O, Benros ME, Gasse C. C-reactive protein and white blood cell levels in schizophrenia, bipolar disorders and depression - associations with mortality and psychiatric outcomes: a population-based study. Eur Psychiatry. 2017;44:164-172. doi:10.1016/j.eurpsy.2017.04.012 
33. Özdin S, Böke Ö. Neutrophil/lymphocyte, platelet/lymphocyte and monocyte/lymphocyte ratios in different stages of schizophrenia. Psychiatry Res. 2019;271:131-135. doi:10.1016/j. psychres.2018.11.043

34. Fu Y, Liu Q, Anrather J, Shi F-D. Immune interventions in stroke. Nat Rev Neurol. 2015;11(9):524-535. doi:10.1038/nrneurol.2015.144

35. Levine DA, Galecki AT, Langa KM, et al. Trajectory of cognitive decline after incident stroke. JAMA. 2015;314(1):41-51. doi:10.1001/ jama.2015.6968

36. Urday S, Kimberly WT, Beslow LA, et al. Targeting secondary injury in intracerebral haemorrhage-perihaematomal oedema. Nat Rev Neurol. 2015;11(2):111-122. doi:10.1038/nrneurol.2014.264

37. Beez T, Steiger H-J, Etminan N. Pharmacological targeting of secondary brain damage following ischemic or hemorrhagic stroke, traumatic brain injury, and bacterial meningitis - a systematic review and meta-analysis. BMC Neurol. 2017;17(1):209. doi:10.1186/ s12883-017-0994-z

38. Walter HL, Walberer M, Rueger MA, et al. In vivo analysis of neuroinflammation in the late chronic phase after experimental stroke. Neuroscience. 2015;292:71-80. doi:10.1016/j. neuroscience.2015.02.024

39. Ramirez K, Fornaguera-Trías J, Sheridan JF. Stress-induced microglia activation and monocyte trafficking to the brain underlie the development of anxiety and depression. Curr Top Behav Neurosci. 2017;31:155-172. doi:10.1007/7854_2016_25

40. Wohleb ES, McKim DB, Sheridan JF, Godbout JP. Monocyte trafficking to the brain with stress and inflammation: a novel axis of immune-to-brain communication that influences mood and behavior Front Neurosci. 2014;8:447. doi:10.3389/fnins.2014.00447

41. Kratofil RM, Kubes P, Deniset JF. Monocyte conversion during inflammation and injury. Arterioscler Thromb Vasc Biol. 2017;37 (1):35-42. doi:10.1161/ATVBAHA.116.308198
42. Maes M, Smith R, Christophe A, et al. Lower serum high-density lipoprotein cholesterol (HDL-C) in major depression and in depressed men with serious suicidal attempts: relationship with immune-inflammatory markers. Acta Psychiatr Scand. 1997;95 (3):212-221. doi:10.1111/j.1600-0447.1997.tb09622.x

43. Tedders SH, Fokong KD, McKenzie LE, Wesley C, Yu L, Zhang J. Low cholesterol is associated with depression among US household population. J Affect Disord. 2011;135(1-3):115-121. doi:10.1016/j. jad.2011.06.045

44. Nakao M, Yano E. Relationship between major depression and high serum cholesterol in Japanese men. Tohoku J Exp Med. 2004;204 (4):273-287. doi:10.1620/tjem.204.273

45. Ledochowski M, Murr C, Sperner-Unterweger B, Neurauter G, Fuchs D. Association between increased serum cholesterol and signs of depressive mood. Clin Chem Lab Med. 2003;41 (6):821-824. doi:10.1515/CCLM.2003.124

46. Rogacev KS, Zawada AM, Hundsdorfer J, et al. Immunosuppression and monocyte subsets. Nephrol Dial Transplant. 2015;30 (1):143-153. doi:10.1093/ndt/gfu315

47. Zhang J, Chen W, Fang L, et al. Increased intermediate monocyte fraction in peripheral blood is associated with nonalcoholic fatty liver disease. Wien Klin Wochenschr. 2018;130(11-12):390-397. doi:10.1007/s00508-018-1348-6

48. Petrone AB, Eisenman RD, Steele KN, Mosmiller LT, Urhie O, Zdilla MJ. Temporal dynamics of peripheral neutrophil and lymphocytes following acute ischemic stroke. Neurol Sci. 2019;40 (9):1877-1885. doi:10.1007/s10072-019-03919-y
Neuropsychiatric Disease and Treatment

\section{Publish your work in this journal}

Neuropsychiatric Disease and Treatment is an international, peerreviewed journal of clinical therapeutics and pharmacology focusing on concise rapid reporting of clinical or pre-clinical studies on a range of neuropsychiatric and neurological disorders. This journal is indexed on PubMed Central, the 'PsycINFO' database and CAS, and is the official journal of The International Neuropsychiatric Association (INA). The manuscript management system is completely online and includes a very quick and fair peer-review system, which is all easy to use. Visit http://www.dovepress.com/testimonials.php to read real quotes from published authors. 